\title{
HIPAA Update: Standards for Health Care Electronic Transactions Finalized
}

\author{
Erin Brisbay McMahon, JD
}

The Department for Health and Human Services (HHS) has issued the final rule that will govern electronic exchanges of financial and administrative information in the health care industry. About 400 different formats currently exist for electronic health care claims. Once compliance with this rule is required (October 2002 for most health care entities to which the rule applies), a physician will be able to submit an electronic claim in the standard transaction format to virtually any health plan in the United States and the health plan will have to accept it.

Under the rule, an electronic transaction involves information exchanges between two parties to carry out financial or administrative activities related to health care. Thus, health plans will be able to pay physicians, authorize services, certify referrals, and coordinate benefits using a standard electronic format for each transaction. Conflicting state laws will be superseded by the standards, although HHS is developing an exception process pursuant to HIPAA.

HIPAA required HHS to adopt data and format standards, if possible, that were developed by private sector standards development organizations accredited by the American National Standards Institute (ANSI). When conducting a transaction covered by the rule, physicians are required to use applicable medical data code sets as specified in the implementation specification that is valid at the time the health care is furnished. Local and proprietary codes currently used by health plans can no longer be used in electronic transactions governed by the rule after the compliance date (October 16, 2002, except for small health plans, which have until October 16, 2003).

This summary of the Standards for Electronic Transactions should not be construed as legal advice or an opinion on specific situations. Please consult an attorney concerning your compliance with HIPAA and the regulations promulgated thereunder.

Keywords: HIPAA update, Health Insurance Portability Act., electronic transactions, electronic standards, health claims, health care claringhouses
The Department for Health and Human Services (HHS) has issued the final rule that will govern electronic exchanges of financial and administrative information in the health care industry. The standards for health care electronic transactions were published in the August 17, 2000 edition of the Federal Register and can be found at http://aspe.os.dhhs.gov/admnsimp. HHS was required to adopt the standards pursuant to the administrative simplification provisions in the Health Insurance Portability and Accountability Act of 1996 (HIPAA). Congress and the regulators hope that the standards will simplify electronic transactions and result in cost savings throughout the industry. According to HHS, about 400 different formats currently exist for electronic health care claims. Once

From the law firm of HargroveBaker, PSC, Lexington, Kentucky. Ms. McMahon is an attorney at HargroveBaker. Address correspondence: Erin Brisbay McMahon, Esq., HargroveBaker, PSC, 2800 Lexington Financial Center, Lexington, KY 40507. Email: EMcMahon@HargroveBaker.com compliance with this rule is required (October 2002 for most health care entities to which the rule applies), a physician will be able to submit an electronic claim in the standard transaction format to virtually any health plan in the United States and the health plan will have to accept it.

The rule applies to health care plans and health care clearinghouses. Health care plans include most private sector health plans that provide or pay the cost of medical care (e.g., managed care organizations), and all government health plans (including Medicare and Medicaid). Health care clearinghouses are public or private entities that either (1) take information received from another entity (e.g., a physician group practice) in a nonstandard format or containing nonstandard data content and convert it into a standard transaction or standard data elements for a receiving entity (e.g., a health plan) or (2) receive a standard transaction from another entity (e.g., a health plan) and convert it into nonstandard format or nonstandard data content for a receiving entity (e.g., a physician group 
practice). The rule also applies to all health care providers who transmit any health information in electronic form in connection with a transaction covered by the rule.

\section{HEALTH CARE TRANSACTIONS SUBJECT TO THE RULE}

Under the rule, an electronic transaction involves information exchanges between two parties to carry out financial or administrative activities related to health care. Transactions subject to the rule include the following types of electronic data interchange:

- Health claims and equivalent encounter information

- Enrollment and disenrollment in a health plan

- Eligibility for a health plan

- Health care payment and remittance advice

- Health plan premium payments

- Health claim status

- Referral certification and authorization

- Coordination of benefits

Thus, health plans will be able to pay physicians, authorize services, certify referrals, and coordinate benefits using a standard electronic format for each transaction. Physicians also will be able to use a standard format to determine a patient's eligibility for insurance coverage, file a claim, ask about the status of a claim, request authorizations for services or specialist referrals, and receive electronic remittances to post receivables. HHS will eventually adopt standards for claims attachments and the first report of injury.

Conflicting state laws will be superseded by the standards, although HHS is developing an exception process pursuant to HIPAA. Specific exceptions to the standards may also be allowed for entities wishing to test (1) a modification to the existing standard or (2) a different standard.

\section{STANDARDS}

HIPAA required HHS to adopt data and format standards, if possible, that were developed by private sector standards development organizations accredited by the American National Standards Institute (ANSI). ANSI Accredited Standards Committee (ASC) X12N standards, Version 4010, were adopted for all transactions except retail pharmacy transactions, which will be governed by the National Council for Prescription Drugs Programs (NCPDP) standards, the NCPDP Telecommunications Standard Format Version 5.1 and NCPDP Batch Standard Version 1.0.

When conducting a transaction covered by the rule, physicians are required to use applicable medical data code sets as specified in the implementation specification that is valid at the time the health care is furnished. Under the rule, a "code set" is any set of codes used for encoding data elements, such as tables of terms, medical concepts, medical diagnostic codes, or medical procedure codes. The standard medical data code sets that must be used to code standard transactions are as follows:

- International Classification of Diseases, 9th Edition, Clinical Modification, (ICD-9-CM), Volumes 1 and 2 (including The Official ICD-9CM Guidelines for Coding and Reporting), as maintained and distributed by HHS, for the following conditions:

- Diseases.

- Injuries.

- Impairments.

- Other health problems and their manifestations.

- Causes of injury, disease, impairment, or other health problems.

- International Classification of Diseases, 9th Edition, Clinical Modification, Volume 3 Procedures (including the Official ICD-9-CM Guidelines for Coding and Reporting), as maintained and distributed by HHS, for the following procedures or other actions taken for diseases, injuries, and impairments on hospital inpatients reported by hospitals:

- Prevention.

- Diagnosis.

- Treatment.

- Management.

- National Drug Codes (NDC), as maintained and distributed by HHS, in collaboration with drug manufacturers, for the following:

- Drugs.

- Biologics.

- Code on Dental Procedures and Nomenclature, as maintained and distributed by the American Dental Association, for dental services. 
- The combination of Health Care Financing Administration Common Procedure Coding System (HCPCS), as maintained and distributed by HHS, and Current Procedural Terminology, Fourth Edition (CPT-4), as maintained and distributed by the American Medical Association, for physician services and other health care services. These services include, but are not limited to, the following:

- Physician services.

- Physical and occupational therapy services.

- Radiologic procedures.

- Clinical laboratory tests.

- Other medical diagnostic procedures.

- Hearing and vision services.

- Transportation services including ambulance.

- The Health Care Financing Administration Common Procedure Coding System (HCPCS), as maintained and distributed by HHS, for all other substances, equipment, supplies, or other items used in health care services. These items include, but are not limited to, the following:

- Medical supplies.

- Orthotic and prosthetic devices.

- Durable medical equipment.

Local and proprietary codes currently used by health plans can no longer be used in electronic transactions governed by the rule after the compliance date (October 16, 2002, except for small health plans, which have until October 16, 2003). The Implementation Guides and Data Dictionaries for Transactions Standards can be downloaded from http://aspe.os.dhhs.gov/admnsimp.

\section{CLEARINGHOUSES}

Physicians may choose to use a business associate (a person who performs an activity falling under the rule on behalf of the physician), including a health care clearinghouse, to conduct transactions covered by the rule. However, the physician must contractually require the business associate (1) to comply with all applicable requirements of the rule and (2) to insist via written contract that the business associate's agents and subcontractors comply with the rule. Health care clearinghouses may prove useful to physicians who prefer to store data in a format that does not translate into a standard transaction.

\section{INTRACORPORATE TRANSACTIONS}

When conducting electronic transactions within corporate boundaries, two questions must be asked to determine if a standard format must be used:

- Is the transaction initiated by a health plan, clearinghouse, or provider subject to the rule or its business associate? If not, the standard need not be followed.

- Is the transaction one for which the Secretary has adopted a standard? If so, the standard has to be used. If not, then the standard need not be followed. For purposes of this question, the definitions of the transactions themselves, as stipulated in subparts K-R of the regulation, must be consulted to determine if the Secretary has adopted a standard for the transaction.

\section{MODES OF TRANSMISSION}

The rule applies to transactions sent over the Internet. However, a physician using a "direct data entry" process, where data are directly keyed into a health plan's computer using dumb terminals or computer browser screens, must comply with the data requirements of the applicable standard, but need not comply with the format portion of the standard.

\section{HEALTH CARE CLAIMS}

The rule does not require physicians to submit claims transactions electronically, but all transactions submitted electronically must comply with the standards. For example, a physician could send an electronic health care claim standard transaction for Patient A to health plan Z, and could also send a paper claim for patient $B$ to health plan Z. The same physician could send an electronic health care claim standard transaction to health plan $\mathrm{S}$ and send paper claims to health plan $\mathrm{T}$.

Under HIPAA, health plans subject to the rule must accept the standard claim submitted electronically. They cannot:

- require physicians to make changes or additions to the standard claim;

- delay or reject a transaction, or attempt to adversely affect a physician practice or the transaction, because the transaction is a standard one;

- reject a standard transaction on the basis that it 
contains data elements not needed or used by the health plan; or

- offer incentives for physicians to conduct a transaction covered by the rule as a direct data entry.

Further, health plans that operate as clearinghouses or that require a physician to use a clearinghouse to receive, process, or transmit a standard transaction cannot charge fees or costs in excess of the fees or costs for normal telecommunications that a physician practice incurs when it directly transmits or receives a standard transaction to or from a health plan.

Until a standard is adopted for electronic health claims attachments (by February 2001) and compliance is required with that standard, health plans can continue to require health claim attachments to be submitted on paper.

Health plans may continue to provide physicians with information regarding their processing or adjudication policies. However, this information cannot be used to modify a standard adopted by HHS and cannot include:

- instructions to change the definition, data condition, or use of a data element or segment in a standard;

- requests for data elements or segments not in the maximum defined data set in the applicable implementation guide;

- requests for codes or data elements that are either marked "not used" in the implementation specifications or are not in the standard's implementation specifications; or

- rules that change the meaning or intent of a standard's implementation specifications.

Similarly, provider contracts with health plans cannot provide for any of the above. This means that physicians choosing to engage in electronic transactions covered by the rule must review their provider contracts with health plans and revise them so that renewals of the contracts occurring on and after October 16, 2002 state that electronic standard transactions must comply with the regulation.

It is important to note that while health plans covered by the rule must accept and process any claim that meets the national standard, they do not have to pay the claim if it is not for a covered service. HHS gives the following example: Medicare currently requires providers to bill for certain services only in specified circumstances. In October 2002, however, Medicare will have to process all claims that meet HIPAA specifications. This does not mean that Medicare has to change a payment policy that denies coverage for cosmetic face lifts; if a claim for a face lift for cosmetic purposes is received after the compliance date, Medicare will have to process the bill, but will not have to pay the claim if its payment policy then in force denies such a claim as a noncovered service.

\section{ENFORCEMENT AND IMPLEMENTATION}

HIPAA gives the Secretary of HHS the authority to impose monetary penalties for failure to comply with a standard. The Secretary is required by statute to impose penalties of not more than $\$ 100$ per violation on any person or entity who fails to comply with a standard. However, the total amount imposed on any one person in each calendar year cannot exceed $\$ 25,000$ for violations of one requirement. The Secretary will eventually adopt a regulation addressing specific enforcement procedures.

In an HHS press release dated August 11, Secretary Shalala cautioned that this rule was released under the assumption that, by the compliance date, HHS' now proposed regulation on privacy of medical records (November 3, 1999 Federal Register) will also be in effect, or that Congress will have enacted such protections. The privacy rule is expected to be issued as a final rule before the November 2000 election. If such privacy protections are not in place by October 16, 2002, HHS has said it will seriously consider suspending or withdrawing the transaction regulation, pending appropriate privacy protections.

Additional information on the administrative simplification regulations of HIPAA, as well as a "Frequently Asked Questions" section on the electronic transactions standards rule, can be found at http://aspe.os.dhhs.gov/ admnsimp, which can be accessed directly or through the HCFA website: http://www.hcfa.gov/medicare/edi/ hipaaedi.htm. 